Euskal ikerketen aldizkaria | Revue d'études basques |

Revista de estudios vascos | Basque studies review

$12 \mid 2008$

Numéro XII

\title{
L, M [texte intégral]
}

L.A. Latina America, L.A.B. Langile abertzaleen batzordeak, L.E.M. [Lycée d'Etat mixte] de Marracq, La Bastide-Clairence, Label auto, Laborari gazte, Laborari-Lo Païsan [puis Laborari], Lagunt eta maita

\section{OpenEdition}

\section{Journals}

Édition électronique

URL : http://journals.openedition.org/lapurdum/1650

DOI : 10.4000/lapurdum. 1650

ISSN : 1965-0655

Éditeur

IKER

Édition imprimée

Date de publication : 1 février 2008

Pagination : 114-129

ISBN : 978-2-86781-617-8

ISSN : $1273-3830$

Référence électronique

« L, M [texte intégral] », Lapurdum [En ligne], 12 | 2008, mis en ligne le 24 mai 2010, consulté le 05 mai 2019. URL : http://journals.openedition.org/lapurdum/1650 ; DOI : 10.4000/lapurdum.1650

Ce document a été généré automatiquement le 5 mai 2019.

Poitelon J.C. | IKER 


\section{L, M [texte intégral]}

L.A. Latina America, L.A.B. Langile abertzaleen batzordeak, L.E.M. [Lycée d'Etat mixte] de Marracq, La Bastide-Clairence, Label auto, Laborari gazte, Laborari-Lo Païsan [puis Laborari], Lagunt eta maita

1 L.A. Latina America. Revue des deux océans. Annuel. automne 1996 - automne 1997 (nº 1-2). - Biarritz, Paris, $24 \times 16 \mathrm{~cm}$ En français

2 * L.A.B. Langile abertzaleen batzordeak. Barneko horria [puis orria]. Irrégulier. [sept. $\left.2000\left(n^{\circ} 1\right)\right] \rightarrow$. [Bayonne], $30 \times 21 \mathrm{~cm}$

En [sept. 2000] a paru sous le titre: L.A.B. Langile abertzaleen batzordeak. Azken berriak, en une seule éd., bilingue; à partir de nov. 2000 ( $\left.n^{\circ} 2\right)$ a paru en 2 éd.: éd. basque et éd. française

$3 \quad$ * L.E.M. [Lycée d'Etat mixte] de Marracq. Informations. [...]. - [Bayonne], 30 x $21 \mathrm{~cm}$ Paraissait en 1977. - En français

4 La Bastide-Clairence. Vie municipale voir Vie municipale

5 Label auto. Bi-mensuel gratuit d'annonces auto-moto \{professionnelles\}. Les spécialistes [ puis Le spécialiste] de l'occasion. $1^{\text {er }}$ oct. - 16 nov. 1992 (n¹-3) [?]. - Anglet, 27 x 19 cm En français

Laborari gazte. Irrégulier. 1977 - 1978 [?]. - [Saint-Jean-Pied-de-Port], 30 x $21 \mathrm{~cm}$

En basque et français. - Publ. par: Centre des jeunes agriculteurs de Garazi [Saint-Jean-Piedde-Port]

Laborari-Lo Païsan [ puis Laborari]. Mensuel [ou Bimensuel puis Hebdomadaire] d'information des paysans basques et béarnais [puis Hebdomadaire d'informations des paysans du Pays basque]. 27 nov. $1982\left(\mathrm{n}^{\circ} 1\right) \rightarrow$. Saint-Jean-Pied-de-Port, $32 \times 23$ puis $40 \times 31$ puis $30 \times 21$ puis $32 \times 23 \mathrm{~cm}$

La 2e partie du titre ("Lo Païsan ») figure en petits caractères à partir de févr. 1992 puis disparaît en 2001. - En français; quelques textes basques. - Publ. par: Euskal herriko laborarien batasuna (E.L.B.) [Saint-Jean-Pied-de-Port]. - Le $n^{\circ} 3$ (janv. 1983) a paru en 2 éd.: éd. Pays basque et éd. Béarn; l'éd. unique porte ensuite jusqu'en 1993 la mention: éd. Pays basque Lagunt eta maita voir Amicale des Basques de Pau...

9 Lahonce. Bulletin municipal voir Lehuntzako berriak 
Lahonce autrement voir Information (L') autrement

Landazaharetik Pekoberhorat. Bulletin municipal d'Amendeuix-Oneix. Annuel. 1989-1994. - Amendeuix-Oneix, 30 x $21 \mathrm{~cm}$

En français. - Devient: Amenduze-Unaso... Bulletin municipal

Landes-Pyrénées-vélo. avr. 1985 (n¹) [?]. - Ciboure, 30 x $21 \mathrm{~cm}$

En français

Langile abertzaleen batzordeak... voir L.A.B...

14 Lapurdum. Revue annuelle du Centre de recherches sur la langue basque et l'expression en langue basque [puis du Centre de recherches sur la langue et les textes basques puis du Centre de recherches Iker]... et du Département interuniversitaire d'études basques (Faculté pluridisciplinaire de Bayonne). oct. 1996 (vol. 1) $\rightarrow$. Bayonne, 24 x $16 \mathrm{~cm}$ Avec un n'spécial de 1999 intitulé: Lettres (Les) basques au temps d'Axular (1600 - 1650). Actes du colloque... - Le sous-titre varie; sous-titre de la couv.: Etudes basques puis Euskal ikerketen aldizkaria. Revue d'études basques. Revista de estudios vascos. Basque studies review. - En français avec quelques textes basques, ou en basque et français

Largenté. \{Le livre de l'année\}. Annuel. 1993/94 - 1994/95. 1996/97 $\rightarrow$. Bayonne, $30 \times 21 \mathrm{~cm}$

De 1996/97 à 1998/99 a paru sous le titre: Livre (Le) de l'année Largenté. - En français. - Publ. par: Largenté. Ecole - collège - lycée [Bayonne]

\section{séminaire de Larressore-Belloc-Ustaritz. Annuel. 1996/97 $\rightarrow$. Ustaritz, 30 x $21 \mathrm{~cm}$}

Le sous-titre varie et disparait. - En français. - Publ. par: Association amicale des anciens élèves du Petit séminaire de Larressore, Belloc et Ustaritz [Ustaritz]. - Suite de: Bulletin de l'Association des anciens élèves (Larresore, Bel-loc et Ustaritz)

Latina America voir L.A.

Lauburu. \{Bulletin d'informations\} [puis Bulletin d'information de l'association Lauburu]. Irrégulier ou trimestriel. [mars 1978] - juin 2001 (n¹-75). - Saint-Jean-de-Luz puis Bayonne, $30 \times 21 \mathrm{~cm}$

Avec un nspécial de juin 1985. - De 1978 à 1980 certains nºnt paru sous le titre: Bulletin Lauburu. - En français; quelques textes basques dans les derniers $n^{\circ}$. - Publ. par: Lauburu [Bayonne]. - Remplacé par: Lauburu. Lettre aux adhérents et aux amis...

$19{ }^{*}$ Lauburu. Lettre aux adhérents et aux amis [puis Lettre d'information] de l'association Lauburu. Irrégulier. juil. $2003\left(\mathrm{n}^{\circ} 3\right) \rightarrow$. Bayonne, 30 x $21 \mathrm{~cm}$

2 circulaires auraient précédé le $n^{\circ} 3$ de la lettre. - En français. - Remplace: Lauburu. Bulletin d'informations

20 Lauka. C.F.D.T. Enseignement privé Pays basque [puis C.F.D.T. Pays basque. Syndicat Enseignement privé puis C.F.D.T. Pays basque. Syndicat Formation et enseignement privés]. Mensuel puis irrégulier. oct. 1992 (n¹) - juin 2003 [?]. - [Bayonne], $26 \times 18$ ou $30 \times 21 \mathrm{~cm}$

Le $n^{\circ} 1$ ne porte pas encore le titre. - Le sous-titre varie. - En français

21 Lauzka. Euskal kultur erakundeko informazio hostoa [puis Euskal kultur erakundearen berripapera]. Lettre d'information de l'Institut culturel basque. Irrégulier. juil. - sept. 1994 (n6-7). févr. 1995 - févr. 1997 (n³-8). - Ustaritz, 30 x $21 \mathrm{~cm}$

En basque et français. - Suite de: Institut culturel basque... Lettre d'information. - Devient: Leihotik 
Lehenik Senpere... voir Saint-Pée d'abord...

Lehuntzako berriak. Semestriel. $1977\left(n^{\circ} 1\right)$ - [...]. [...] - janv. 1995. sept. 1995 - juil. 2000 (nº 1-10) [?]. $2001 \rightarrow$. Lahonce, $30 \times 21 \mathrm{~cm}$

Titre français de 1977 à [...] : Information. Lahonce puis de sept. 1995 à juil. 2000 : Lahonce. Bulletin municipal puis Bulletin municipal de Lahonce. - De [...] à janv. 1995 et à partir de 2001 a paru sous le seul titre: Bulletin municipal. Lahonce ou Lahonce. Bulletin municipal. En français

* Leihoa. \{Le\} bulletin des Amis d'Errobi [puis d'Errobiko festibala]. [févr. - juil. 1998] (nº 1-5) [?]. - Itsasu, $30 \times 21 \mathrm{~cm}$

En français; quelques textes basques. - Publ. par: Amis (Les) d'Errobiko festibala [Itxassou]

Leihotik. Euskal kultur erakundearen berripapera. Bulletin d'information [puis d'information bimestriel] de l'Institut culturel basque. Bimestriel puis trimestriel. janv./ févr. $1998\left(\mathrm{n}^{\circ} 1\right) \rightarrow$. Ustaritz, 34 x $24 \mathrm{~cm}$ En basque et français. - Suite de: Lauzka

Lema. Euskadi Europan. Mensuel. févr. $1997\left(n^{\circ} 1\right) \rightarrow$. Baiona, 30 x 21 ou 34 x $24 \mathrm{~cm}$ En français; quelques textes basques. - Publ. par: Eusko alderdi jeltzalea (E.A.J.); en français: Parti nationaliste basque (P.N.B.) [Bayonne]

Léo-cancans. Tous les 4 mois. [nov. 1993] - févr. 2002. - Bayonne, 30 x $21 \mathrm{~cm}$ En français. - Publ. par: Club Léo Lagrange de Bayonne [Bayonne]. - Reprend les éléments de: Shuss

Lerroz lerro voir Arrapitz lerroz lerro

Lettre (La). Nouvelles officielles du B.T.P. 40, 64, 65. Lettre hebdomadaire d'informations du B.T.P. 11 sept. 1995 ( $\left.{ }^{\circ} 1\right)$ - [...]. - Anglet puis Biarritz, 30 x $21 \mathrm{~cm}$ Paraissait encore en 2000 [?]. - En français. - Il a paru du 12 févr. 1997 à août 1998 [?] une autre éd. pour la Gironde. - Pour le bulletin mensuel voir Nouvelles officielles du B.T.P. 64

Lettre (La) Atlantica - Séguier. Trimestriel puis mensuel. mars 1999 - déc. 2001 (n¹-20). Biarritz, Paris puis Anglet, Paris, 30 x $21 \mathrm{~cm}$ En français. - Publ. par: Atlantica éditions [Biarritz, Paris]

Lettre aux électeurs. [déc. 1976 - mars 1977] (n¹-8) [?]. - [Mougerre], 30 x $21 \mathrm{~cm}$ En français. - Publ. à l'occasion des élections municipales de mars 1977 pour soutenir la «Liste d'union de la gauche ». - Devient: Union (L') de la gauche « informe »

Lettre aux maires. Landes - Pyrénées-Atlantiques. Semestriel. févr. 1991 - janv. 1995 ( ${ }^{\circ}$ 1-9) [?]. - Bayonne, 30 x $21 \mathrm{~cm}$ En français. - Publ. par: Electricité de France - Gaz de France services Sud-Aquitaine [Bayonne]. - Voir aussi Tam-tam 640

Lettre aux oblats de Belloc voir Abbaye N.-D. de Belloc. Lettre aux oblats Lettre (La) d'Activités en Pays basque voir Activités en Pays basque

Lettre (La) d'Alain Lamassoure. Député des Pyrénées-Atlantiques. Député au Parlement européen. Lettre trimestrielle. sept. 1990 - juil. 1991 (n¹-3)[?]. [...]. - Bayonne, 30 x $21 \mathrm{~cm}$ Avec un n'[0] d'[avr./mai 1990]. - A paru en nov. 2001. - En français

Lettre (La) d'Anglet. janv. - déc. 1988 (n¹-3). - Anglet, 30 x $21 \mathrm{~cm}$

Publ. par la Ville d'Anglet. - En français. - Suite de: Anglet. La lettre municipale. - Paraît parallèlement à: Anglet. Revue municipale... 
43

Lettre (La) d'Euskadi. Bulletin[puis Mensuel] d'information sur la Communauté autonome basque. Mensuel. mars $1997\left(\mathrm{n}^{\circ} 1\right) \rightarrow$. Bayonne, 30 × $21 \mathrm{~cm}$ En français

Lettre (La) d'information du Syndicat intercommunal d'équipement et d'aménagement de Saint-Jean-de-Luz et de Ciboure [puis de Saint-Jean-de-Luz, Ciboure et Urrugne]. Bulletin semestriel. oct. 1999 - juil. 2001 ( $\left.{ }^{\circ} 1-4\right)$. - Saint-Jean-de-Luz, $30 \times 21 \mathrm{~cm}$

En français

Lettre (La) d'infos de la C.P.A.U. Pays basque, Conférence permanente d'aménagement et d'urbanisme. janv. 1997 ( $\left.\mathrm{n}^{\circ} 0\right)$. - Bayonne, 30 x $21 \mathrm{~cm}$ En français

Lettre d'union de la gauche voir Union (L') de la gauche « informe »

* Lettre (La) de B.C.N. [Biarritz communication nouvelle]. Mensuel. 1987 - 1991 [?]. Biarritz, $30 \times 21 \mathrm{~cm}$

En français

$$
10 \text { janv. } 1976\left(n^{\circ} 1\right) \text { - [...]. - Biarritz,30 x } 22 \mathrm{~cm}
$$

Paraissait encore en août/sept. 1982 (n³1). - En français

44 Lettre (La) de Biarritz autrement - Biarritz [ou Biarritze] bestelakoa. Mensuel. juin 1993 - juin/juil. 1996 (n¹-36). juin 2001 - oct. 2002 (n.s. n¹-4). - Biarritz, 30 x $21 \mathrm{~cm}$ En français; en 2001 et 2002 quelques textes basques. - Publ. par: Biarritz autrement; en basque: Biarritz bestelakoa [Biarritz]. - Suite de: Biarritz autrement

Lettre (La) de Côte basque-rassemblement voir Côte basque - rassemblement

Lettre (La) de Franz Duboscq. Trimestriel. déc. 1989 - août 1992 (n¹-12). - AroueIthorrots-Olhaiby, $30 \times 21 \mathrm{~cm}$

En français

47 Lettre (La) de Jean Espilondo, votre conseiller général. Lettre d'information du canton d'Anglet-Nord. Blancpignon, Brises-Lames, Butte-aux-Cailles, Camiade, Chambred'Amour... mai 1999 - déc. 2000 (n¹-[2]). févr. 2004 ( $n^{\circ} 3$ ). - Bayonne puis Anglet, $30 \times 21 \mathrm{~cm}$

En mai 1999 a paru sous le titre: Mot (Le) de Jean Espilondo, votre conseiller général. - Titre courant: Lettre (La) de votre conseiller général, Jean Espilondo. - En français

48 Lettre (La) de Jean Espilondo, votre député. Lettre d'information de la cinquième circonscription des Pyrénées-Atlantiques. Anglet, Arancou, Ayherre, Bardos, Bayonne... mai 1999 (n¹). - Bayonne, 30 x $21 \mathrm{~cm}$ Titre courant: Lettre (La) de votre député, Jean Espilondo. - En français

* Lettre (La) de Jean Grenet. La lettre d'information du député de la $\mathrm{V}^{\mathrm{e}}$ circonscription des Pyrénées-Atlantiques. Irrégulier. [juin 2003] $\rightarrow$. Bayonne, 30 x $22 \mathrm{~cm}$ En français

Lettre (La) de l'immobilier. \{Pour\} acheter, vendre ou louer au Pays basque. Mensuel. janv. $1996 \rightarrow$. Biarritz puis Saint-Martin-d'Arrossa, 33 x 24 cm En français 
51 Lettre (La) de l'U.D.F. Bulletin d'information de la Fédération U.D.F. des PyrénéesAtlantiques (Section Pays basque). [1980] (n¹) - [...]. - Biarritz, 30 x $21 \mathrm{~cm}$

Porte en tête: Union pour la démocratie française. - En français

Lettre (La) de la 5ème. Journal d'informations du R.P.R. Irrégulier. 1999 [?] - févr. 2002 (nº 11). - Bayonne puis Anglet, $30 \times 21 \mathrm{~cm}$

En français. - Publ. par: Rassemblement pour la République. $5^{\mathrm{e}}$ circonscription des Pyrénées-Atlantiques [Bayonne]. - Reprend les éléments de: Echo (L') des cantons. - Certains élémnets repris par: Trait d'union. Journal d'information de l'U.M.P...

Lettre (La) de Leïhorra. Annuel. 1996-1998 (n¹-3). - Ciboure, 30 x $21 \mathrm{~cm}$

En français. - Publ. par: Amis (les) de Leïhorra [Ciboure]

Lettre (La) de Max Brisson. Conseiller municipal de Biarritz, conseiller régional d'Aquitaine [puis Conseiller général et municipal de Biarritz]. oct. 1997 - juin 2000 (n¹-3). - Biarritz, $30 \times 21 \mathrm{~cm}$

En français. - En juin 2000 même texte que le n¹1 de même date de: Echos (Les) de Biarritz et du Pays basque

55 * Lettre (La) de Saint-Hubert. Bulletin de liaison du St-Hubert Côte basque. oct. 2003 ( ${ }^{\circ}$ 1) $\rightarrow$. [Anglet], $30 \times 21 \mathrm{~cm}$

En français. - Publ. par: Association intercommunale de chasse Saint-Hubert Côte basque [Anglet]

Lettre (La) de votre conseiller général, Jean Espilondo voir Lettre (La) de Jean Espilondo, votre conseiller général

Lettre (La) de votre député, Jean Espilondo voir Lettre (La) de Jean Espilondo, votre député

Lettre (La) des adhérents Uhaitza. juin 1999 - janv. 2000 ( $\left.n^{\circ} 1-3\right)$. - Menditte, 30 x $21 \mathrm{~cm}$ En français. - Remplace: Lettre (La) des associations en Soule

Lettre (La) des associations en Soule. 15 déc. 1997 ( $\left.n^{\circ} 1\right)$. - Menditte, 30 x $21 \mathrm{~cm}$ En français; pour l'éd. basque voir Alkarteen kaseta Züberoatik. - Publ. par: Uhaitza [Menditte]. - Remplace: Uhaitza. Bulletin d'information. - Remplacé par: Lettre (La) des adhérents Uhaitza

Lettre (La) des libéraux \{du Pays basque\}. Bulletin d'information locale de l'opposition [puis Bulletin d'information du Parti républicain, Pays basque]. Irrégulier. [...]. janv. 1996 $\left(n^{\circ} 1\right)$ - [...]. - Biarritz, $30 \times 21 \mathrm{~cm}$ Paraissait déjà en 1981. - En français

61 Lettre (La) du C.P.I.E. [Pays basque]. Centre permanent d'initiatives pour l'environnement. Semestriel puis irrégulier. déc. $1998\left(n^{\circ} 1\right) \rightarrow$. Saint-Etienne-de-Baïgorry, $30 \times 21 \mathrm{~cm}$ En français. - Publ. par: Comité Izpegi [Saint-Etienne-de-Baïgorry]

62 * Lettre (La) du C.R.E.A. [...] - [sept./oct.] 2002 (n³). - Bayonne, 30 x $21 \mathrm{~cm}$ En français. - Publ. par: Club des créateurs repreneurs d'entreprises aquitains puis Club des créateurs et des repreneurs d'entreprise aquitains [Bayonne]

Lettre (La) du canton. Saint-Pierre-d'Irube, Lahonce, Mouguerre, Urcuit, Villefranque. Trimestriel. 1988 - août 1994 (n¹-20). - Saint-Pierre-d'Irube, 34 x $24 \mathrm{~cm}$ En français. - Fondé à l'occasion des élections cantonales de sept. 1988 pour soutenir la candidature de Jean-Pierre Destrade 

$\left(n^{\circ} 1-21\right)$. janv. $1998\left(n^{\circ} 22\right) \rightarrow$. Hendaye, 32 × 22 puis $30 \times 21 \mathrm{~cm}$ Avec un $n^{\circ}$ spécial de 1990 intitulé: Spécial Sokoburu. - En français. - Suite de: Hendaye. Bulletin officiel municipal Pyrénées-Atlantiques. Trimestriel. $3^{\mathrm{e}}$ trim. 1998 - [mars/avr. 2000] (n¹-6). - Pau, Bayonne, $30 \times 21 \mathrm{~cm}$

En français. - Publ. par: Ordre national des médecins. Conseil départemental des PyrénéesAtlantiques [Pau, Bayonne]

$$
\text { En français. - Publ. par: Saiak [Arcangues]. - Reprend les éléments de: Itxaropena. - Devient: }
$$
Saiaren luma la Ligue communiste révolutionnaire - Quatrième Internationale. Supplément à Rouge. Irrégulier. 1989 - sept. 1995. - Bayonne, 30 × $21 \mathrm{~cm}$ Le sous-titre varie et disparaît. - En français. - Texte en partie identique à celui du bulletin de même titre publ. à Pau par la Section du Béarn. - Suite de: Zutik! L.K.I.-ren aldizkaria. - Devient: Piment (Le) rouge

Lettres (Les) de l'informatique et des techniques associées. Mensuel. juil. $1989\left(\mathrm{n}^{\circ} 1\right)$ févr. 1990. - Halsou, 30 x $21 \mathrm{~cm}$ En français. - Devient: Provincial (Le) informatique

Levain (Le) voir Altxagarri 
77 Lézarde (La). Bulletin d'informations. [sept. 1997]. - Bayonne, 30 × $21 \mathrm{~cm}$ En français. - Publ. par: Lézards qui bougent [Bayonne]. - A paru parallèlement à: Lézarde (La). Le trimestriel de Lézards qui bougent

Lézarde (La). Le trimestriel de Lézards qui bougent. oct./déc. 1996 - avr./juin 1998. Bayonne, $30 \times 21 \mathrm{~cm}$

En français. - Parallèlement a paru en sept. 1997 : Lézarde (La). Bulletin d'informations. - A partir d'oct./déc. 1997 paraît avec: A l'air libre

79 Liberté. \{Organe de la\} Section de Mauléon du P.C.F. Irrégulier. juin 1977 - mars 1989 ( $\mathrm{n}^{\circ}$ 1-43). mars $1992\left(n^{\circ} 44\right) \rightarrow$. Mauléon, de 30 x 21 à 42 x $30 \mathrm{~cm}$

Avec des suppl. de janv. et mars 1995. - En français. - Publ. par: Parti communiste français. Section de Mauléon [Mauléon]. - Voir aussi Supplément à la revue municipale du P.C.F. L'Elu d'aujourd'hui

80 Licq-Athérey. Bulletin municipal. Irrégulier. [...]. [1989-1990/92] (n¹-2). $1995 \rightarrow$. LicqAthérey, $30 \times 21 \mathrm{~cm}$

Paraissait déjà en [1980] (n4). - Certains nºnt paru sous le titre: Commune de Licq-Athérey. Bulletin municipal \{d'information\}. - En français

81 Lien (Le) [S.G.E.N. - C.F.D.T. du Pays basque] voir Lokarria. Bulletin d'information...

82 Lien A.P.F. [puis Lien]. Trimestriel ou irrégulier. 15 juil. 1954 - 28 déc. 1977 ( $\left.\mathrm{n}^{\circ}[1]-124\right) .15$ févr. 1978 - [1999] (n.s. n¹-88). - Bayonne puis Bayonne, Pau, 30 x 21 puis 24 x $16 \mathrm{~cm}$ Les 2 premiers $n^{\circ}$ ont paru sous le titre: Association des paralysés de France. Délégation des Basses-Pyrénées. - En français. - Publ. par: Association des paralysés de France. Délégation des Basses-Pyrénées [puis des Pyrénées-Atlantiques] [Bayonne puis Pau, Bayonne]

Ligue d'Ile-de-France de pelote basque. Annuel. 1990-1998. - Paris, 21 x $15 \mathrm{~cm}$ En français

Lire et réagir. Union fédérale de consommateurs Que choisir "Pays basque » [puis Bulletin trimestriel \{de l'\}U.F.C. Que choisir Pays basque]. Trimestriel. $4^{\mathrm{e}}$ trim. $1994\left(\mathrm{n}^{\circ} 1\right) \rightarrow$. Bayonne, $30 \times 21 \mathrm{~cm}$ En français Lis ces kutz. \{Le seul et unique fanzine inter-lycées
- mai 2000] (n¹-5). - [Bayonne], 21 x 15 ou $30 \times 21 \mathrm{~cm}$ En français: quelques textes basques. - Rédigé par un groupe de lycéens à la suite d'une grève de nov. 1998

Littoral Atlantic. Un nouveau magazine européen. Una nueva revista europea. Trimestriel. $1^{\mathrm{er}}$ sept. - $1^{\mathrm{er}}$ déc. 1989 (n¹-2) [?]. - Anglet, 30 x $23 \mathrm{~cm}$ En espagnol et français Littoral Sud-Gascogne. Trimestriel. $2^{\mathrm{e}}$ trim. 1976 ( $\left.\mathrm{n}^{\circ} 1\right)$ - 1977 [?]. - Lahonce, 23 x $16 \mathrm{~cm}$ En français stade

Livre (Le) de l'année Largenté voir Largenté. Le livre de l'année

Lokarri. Auñamendi elgartearen agerkaria. Irrégulier. juin $1973\left(n^{\circ} 1\right)$ - [...]. - Bayonne, $30 \times 21 \mathrm{~cm}$ 
Paraissait encore en mai 1980 ( $\left.n^{\circ} 6\right)$. - En français; quelques textes basques. - Publ. par: Auñamendi [Bidarray]

Lokarria. Bulletin d'information du S.G.E.N. - C.F.D.T. Pays basque. Mensuel. mars 1984 févr. 2004 (n¹-198). - Bayonne, 30 x $21 \mathrm{~cm}$

Avec un $n^{\circ} 0$ de janv. 1984 paru sous le titre: Bulletin d'informations du S.G.E.N. - C.F.D.T. Pays basque; avec de nombreux suppl. - Titre français: Lien (Le). - En français. - Publ. par: Syndicat général de l'éducation nationale - C.F.D.T. du Pays basque [Bayonne]. - En sept. 1992 absorbe: S.G.E.N. - C.F.D.T. Pays basque

Lokarria. Lettre d'information du personnel de la société Sokoa. Sokoako langileen informazio gutuna. \{Carta de información del personal de Sokoa\}. Mensuel. janv. 1988 ( $\left.n^{\circ} 1\right)$ $\rightarrow$. Hendaye, $30 \times 21 \mathrm{~cm}$

Le sous-titre varie. - En français; quelques textes basques. - Publ. par: Sokoa [Hendaye]. - Voir aussi Loya et Soko moko

Lortu ala borrokatu. Organe du Parti bonarchiste Donibane - Ziburu [sic]. févr./avr. 1987

[?]. - [Saint-Jean-de-Luz], $30 \times 21 \mathrm{~cm}$

Bulletin politique en forme de « fanzine ». - En français

Louhossoa. Bulletin municipal. Annuel. $1989 \rightarrow$. Louhossoa, 30 x $21 \mathrm{~cm}$

Certains $n^{\circ}$ ont paru sous le seul titre: Bulletin municipal. - En français

Loya. Lettre d'information. $3 n^{\circ}$ par an. déc. $2000\left(n^{\circ} 1\right) \rightarrow$. Hendaye, 30 x $21 \mathrm{~cm}$

En français. - Publ. par: Sokoa [Hendaye]. - Voir aussi Lokarria. Lettre d'information... et Soko moko

Lumière sur l'Adour. \{Bulletin mensuel distribué dans tous les foyers des paroisses de Bayonne, Boucau, Saint-Pierre-d'Irube\}. Mensuel. mars 1963 (n¹) - 1971 [?]. - Bayonne, de $27 \times 21$ à $31 \times 23 \mathrm{~cm}$

Bulletin interparoissial. - Avec de 1964 à 1966 [?] des nspéciaux d'été, communs à plusieurs bulletins paroissiaux de la région, portant le titre: Au soleil de la saison. - En français. - Texte en partie identique à celui de nombreux autres bulletins paroissiaux; cette partie commune est intitulée. Images du mois. - Remplace: Bulletin paroissial de la cathédrale

Lur berri-informations. \{Organe [puis Organe bimestriel] d'information techniques et économiques des agriculteurs de la région de Saint-Palais\}. Mensuel puis bimestriel puis irrégulier. $1^{\mathrm{er}}$ oct. [?] $1965-1^{\mathrm{er}}$ nov. 1977. nov. $1978\left(\mathrm{n}^{\circ} 1\right) \rightarrow$. Saint-Palais puis Aïcirits, $50 \times 33$ puis $56 \times 38$ puis $30 \times 21 \mathrm{~cm}$

En français. - Publ. par: Lur berri [Saint-Palais puis Aïcirits]. - Fait suite à une rubrique publ. dans: Journal (Le) de Saint-Palais, de 1964 au 25 juil. 1965 et qui porte déjà le titre, "Lur berri informations ", à partir du 7 févr. 1965. - Reprend aussi les éléments de: Bulletin intérieur de liaison des organisations professionnelles agricoles de la région de Saint-Palais

Lurraldea. Lettre trimestrielle conjointe, Conseil des élus, Conseil de développement. Trimestriel puis irrégulier. janv. 1996 - déc. 2001 (n¹-14). - Bayonne, 30 x 21 puis 42 x $30 \mathrm{~cm}$ Le sous-titre varie et disparaît. - En français; quelques textes basques. - Publ. par: Conseil des élus du Pays basque et Conseil de développement du Pays basque; en basque: Euskal herriko hautetsien kontseilua et Euskal herriko garapen kontseilua [Bayonne]. - Voir aussi Barnetik

Luz'affaires. Journal gratuit de publicité de l'Union commerciale luzienne et cibourienne. 30 nov. $1989\left(n^{\circ} 1\right)$ [?]. - Boucau, 41 x $29 \mathrm{~cm}$ En français 
- - [Bayonne], $30 \times 21 \mathrm{~cm}$

En français. - Publ. par: Mouvement pour la France. Fédération des Pyrénées-Atlantiques [Billère] européen, \{conseillère régionale d'Aquitaine,\} conseillère municipale de Bayonne. Annuel ou semestriel. 1990-1993. 1995-1996. - Impr. à Bayonne, 30 x 21 cm

En français. - 1993 a donné lieu à une 2e éd., datée 1993/févr. 1994

En janv. 2000 a paru sous le titre: Magazine Hormadi. - En français. - Publ. par: Anglet Hormadi club [Anglet]. - Remplace: Anglet Hormadi club. Hockey sur glace. Saison et Orques (Les)...

110 Magazine (Le) du G.P.I. Adour - Côte basque \{et Hendaye - Pays basque\}. Trimestriel. été 1997 - printemps 2000. - [Biarritz puis Anglet], 30 x $21 \mathrm{~cm}$

En français. - Publ. par: Groupement des professionnels immobiliers (G.P.I.) [Biarritz puis Anglet]. - Suite de: Sélection immobilière

111 Maiatz. \{Literatur aldizkaria\}. Irrégulier. févr. $1982\left(n^{\circ} 1\right) \rightarrow$. Bayonne, 30 x 21 ou 32 x 23 puis $27 \times 21 \mathrm{~cm}$

Avec un suppl. d'avr. 1995 intitulé: Ezkonduko ditugu ?, par Antton Luku. - En basque. - Publ. par: Maiatz [Bayonne] Bulletin annuel d'information. Amicale des marins et anciens marins]. 1994 ( $\left.\mathrm{n}^{\circ} 12\right) \rightarrow$. Anglet, 30 x $21 \mathrm{~cm}$

Avec un suppl. de [1999] intitulé: Un vieux marin raconte... - En français. - Suite de: Gazette (La) des marins 
En français

En français Maule, $30 \times 21 \mathrm{~cm}$ aldi »-zkaria! En français En français

Mairie de Berrogain-Laruns. Bulletin municipal. Irrégulier ou mensuel puis $2 n^{\circ}$ par an. [... ] - 1995 [?]. - Berrogain-Laruns, 30 x $21 \mathrm{~cm}$

Paraissait déjà en 1981. - A partir de déc. 1994 a paru sous le titre: Commune de BerrogainLaruns. Bulletin municipal. - En français

Mairie de Briscous. Lettre d'information voir Briscous. Lettre municipale

Mairie de Halsou. Bulletin municipal [puis Bulletin d'informations municipales. Herriko berriak]. Irrégulier puis annuel. $3^{\mathrm{e}}$ trim. $1995\left(\mathrm{n}^{\circ} 1\right) \rightarrow$. Halsou, 30 x $21 \mathrm{~cm}$

En 1996 et 1997 a paru sous le titre: Bulletin municipal. Mairie d'Halsou. - En français

Mairie de Larressore. Bulletin municipal. Irrégulier puis semestriel puis annuel. 7 juil. $1983\left(\mathrm{n}^{\circ} 1\right) \rightarrow$. Larressore, $30 \times 21 \mathrm{~cm}$

Mairie de Mendionde. Bulletin municipal voirMendionde. Bulletin municipal

Mairie de Souraïde. Bulletin municipal voir Souraïde. Bulletin municipal

Makila (Le). Inédit à Saint-Jean-de-Luz: un bulletin d'information novateur! [puis De gauche et luzien!]. [été 1997] - 1998 (3 nº). - Saint-Jean-de-Luz, 30 x $21 \mathrm{~cm}$

Makila gorria. Xiberoko Batasunaren aldizkaria. Bimestriel. janv./févr. 2003 ( $\left.n^{\circ} 1\right)$ - 2004. -

En basque et français. - Publ. par: Batasuna [Saint-Palais]. - Texte en partie identique à ceux de: Harian ari [Bayonne] et Hor dago [Saint-Palais]

3 Makilas (Les) du golf [puis Makilas (Les) S.A.E. [Société auxiliaire d'entreprises] du golf puis Makilas (Les) Eiffage du golf]. Annuel. 1986 [?] $\rightarrow$. Bassussarry, 31 x $21 \mathrm{~cm}$

En français. - Publ. par: Challenge organisation [Bassussarry]. - Voir aussi News Makilas

4 Mantxut ? Trimestriel. oct. $1991\left(\mathrm{n}^{\circ} 1\right)$ - 1993 [?]. - [Bayonne] [?], 30 x $21 \mathrm{~cm}$

Avec un $n^{\circ} 0$ de mai 1991 et un $n^{\circ}$ spécial de mars 1992 publ. à l'occasion des élections régionales. En basque et français. - Publ. par: Eskualdun gazteria [Bayonne]. - Voir aussi «Gazteen

Mantxut ? Oztibarreko aldizkaria. Irrégulier. janv. $1999\left(n^{\circ} 1\right) \rightarrow$. Izura, 30 x $21 \mathrm{~cm}$ Texte basque et français

Marins (Les) d'Anglet voir Gazette (La) des marins

Marracq-info [puis Marracq-info professeurs]. \{Bulletin du Collège Marracq\} [puis Journal du Collège Marracq]. Mensuel ou irrégulier. févr. $2000\left(n^{\circ} 1\right) \rightarrow$. [Bayonne], 30 x $21 \mathrm{~cm}$

Marracq-info parents. Journal d'information du Collège Marracq pour les parents. Trimestriel. déc. $2001 \rightarrow$. Bayonne, $30 \times 21 \mathrm{~cm}$

Marraka. déc. 1995 ( $\left.n^{\circ} 1\right)$ - 1996. - Donibane Lohizune, 30 x $21 \mathrm{~cm}$ Bulletin politique en forme de « fanzine ». - En basque

Matelauga. Le journal des lycéens \{de Lauga\} [puis Le seul journal qui résiste à Hersant!!]. Irrégulier. [févr. 1984] (n¹) - oct./nov. 1986 (n¹). - [Bayonne], 30 x $21 \mathrm{~cm}$

En français. - Rédigé par des élèves du Lycée Lauga. - Remplacé par: Galaupin (Le) 

puis $32 \times 24 \mathrm{~cm}$

Mattin megadenda. $4 n^{\circ}$ par an. nov. $2004\left(n^{\circ} 14\right) \rightarrow$. Baiona, $30 \times 21 \mathrm{~cm}$ En basque et français. - Publ. par: Mattin megadenda [Bayonne]. - Suite de: Megazine

Mauléon-Soule. Bulletin municipal. Semestriel puis trimestriel. sept. 1977 - janv. 1983 ( ${ }^{\circ}$ 1-12). août 1983 - janv. 1989 (n¹-23). juil. 1989 - avr. 1995 (n¹-24). automne 1995 - hiver $2000 / 2001\left(n^{\circ} 1-22\right)$. printemps/été $2001\left(n^{\circ} 1\right) \rightarrow$. Mauléon, $30 / 32$ x $23 \mathrm{~cm}$ En français. - Suite de: Mauléon-Soule. Revue municipale

Mauléon-Soule. Revue municipale. 1976. - Mauléon-Soule, 27 x $21 \mathrm{~cm}$ En français. - Devient: Mauléon-Soule. Bulletin municipal

Megazine. \{Il se passe toujours quelque chose à la librairie Mattin megadenda\}. $4 n^{\circ}$ par an. $1^{\mathrm{er}}$ juin 2001 - juil. 2004 (n॰1]-13). - Baiona, 30 x $21 \mathrm{~cm}$ Titre basque: Megahitz. - En basque et français. - Publ. par: Mattin megadenda [Bayonne]. Devient: Mattin megadenda

Megazine. Le journal des jeunes de Biarritz. Irrégulier. été $1997\left(n^{\circ} 1\right) \rightarrow$. Biarritz, 33 x 28 En français. - Publ. par la Ville de Biarritz

Mende berri. Bimensuel puis mensuel. 15 sept. - 15 oct. 1973 ( $\left.n^{\circ} 1-[3]\right)$. avr. - juin 1974 ( ${ }^{\circ}$ 4-6). oct. 1974 ( $\left.\mathrm{n}^{\circ} 7\right)$. - Bayonne, 30 x 21 ou 21 x $15 \mathrm{~cm}$

Avec un suppl. au $n^{\circ} 7$ de [1975]. - En français; quelques textes basques. - Publ. par: Mende berri [Bayonne]. - Pour le suppl., qu'il contient d'avr. à juin 1974, voir Etorki. - Remplacé par: Gernika. Euskal gudua

Mende berri. Organe basque-français des intérêts du Pays basque. nov. 1958 ( $4^{\mathrm{e}}$ année, $\mathrm{n}^{\circ}$ 4 [sic]). - Orègue, $44 \times 30 \mathrm{~cm}$

En basque et français. - Publ. à l'occasion des élections législatives de nov. 1958 pour soutenir la candidature de Jean Errecart

Mendiloriosa [puis Mendiloriosa ttipitua]. \{La montagne créatrice de joie, la montagne qui procure le bonheur\}. Trimestriel. mars $1980\left(\mathrm{n}^{\circ} 1\right) \rightarrow$. Bayonne, $30 \times 21 \mathrm{~cm}$ En français. - Publ. par: Club alpin français. Section Bayonne - Pays basque [Bayonne]

Mendionde. Bulletin municipal. Annuel puis semestriel. [1990-1993] [?]. janv. 1996 (n¹) $\rightarrow$. Mendionde, $30 \times 21$ ou $21 \times 15 \mathrm{~cm}$

Jusqu'en 1993 a paru sous le titre: Mairie de Mendionde. Bulletin municipal; de juin 1996 à janv. 1998 a paru sous le titre: Bulletin municipal \{de\} Mendionde. - Titre basque en janv. 1996 : Herriko etxetik. - En français; quelques textes basques

Mezulari [puis Mezulari Lapurdi]. Journal interparoissial du Labourd. Irrégulier. juin/juil. 1974 - mars/avr. 1989 (n¹-62). - Saint-Martin-d'Arberoue puis Villefranque puis Camboles-Bains puis Cambo-les-Bains, Ustaritz, 26 x 19 puis $27 \times 21 \mathrm{cmEn}$ basque et français. Suite de: Gure artean mezulari

* Mezulari. Parropiako berriak. Infos paroissiales. Irrégulier. févr. 2000 - juil. 2002 (n5). Hasparren, $30 \times 21 \mathrm{~cm}$

Bulletin de la paroisse Bienheureux François d'Ardan [Hasparren]. - En basque et français

Miarritzeko ahatea. Irrégulier. oct. 1978 (n¹) - [...]. - [Biarritz], 21 x $15 \mathrm{~cm}$ Paraissait encore en 1981 ( $\left.n^{\circ} 7\right)$. - En français; quelques textes basques. - Publ. par: Miarritzeko abertzaleen taldea puis Miarritzeko herri taldea [Biarritz]

Mieux vivre à Bidart voir Bidarten hobeki 


\section{$30 \times 21 \mathrm{~cm}$}

En français. - Voir aussi Orec news éléments repris par: Pays basque - Adour

$\rightarrow$. Villefranque, $30 \times 21 \mathrm{~cm}$

En basque et français 10-11) [?]. - Bayonne, 30 x $21 \mathrm{~cm}$ votre conseiller général

1967-1972 [Mil neuf cent soixante-sept - mil neuf cent soixante-douze]. Organe du Comité de soutien de l'action de la $\mathrm{V}^{\mathrm{e}}$ République [puis de l'action du président de la République]. Bimensuel puis irrégulier. 15 janv. 1967 - 11 mai 1974 ( $1^{\mathrm{re}}-8^{\mathrm{e}}$ année, $\left.\mathrm{n}^{\circ} 1-17\right)$ [?]. - Saint-Jean-Pied-de-Port puis Ossès puis Irissarry puis Saint-Palais, de 45 x 28 à 60 x $45 \mathrm{~cm}$ Avec un "Supplément féminin » au nº du 26 févr. 1967. - En français; quelques textes basques. Publ. à l'occasion de diverses élections, notamment à l'occasion des élections législatives de mars 1967, juin 1968 et mars 1973 pour soutenir la candidature de Michel Inchauspé. - En 1977

Milafranga. Herriko berriak. Bulletin d'informations locales. Trimestriel. juin 1983 ( $\left.\mathrm{n}^{\circ} 1\right)$

Bulletin municipal de Villefranque. - En français. - Suite de: Gure Milafranga

de la langue basque. avr. 2002 - janv. 2003 (n¹-4). - Ustaritz, 36 x $26 \mathrm{~cm}$

Miramar-news. Irrégulier. 1994 - automne/hiver 1997/98. - Biarritz, 30 x $21 \mathrm{~cm}$

En français. - Publ. par: Hôtel Miramar [Biarritz]

Mire de barges. Mensuel puis irrégulier. 1997 [?] - mai 1999 (nº-[9]). févr.-mars 2004 (nº

En français. - Publ. par: Rayon bleu, association des élèves du B.T.S. Audiovisuel, au Lycée René Cassin [Bayonne]. - Remplace: Crayon bleu

Miroir (Le) de la Soule \{et de la Haute-Soule\}. \{Défense des intérêts de la vallée\} [puis Défense des intérêts du Pays de Soule, nouvelles du Pays basque et du Barétous puis Défense des intérêts, et informations de la Soule, du Pays basque et du Barétous]. Bimensuel. 11 août $1956\left(\mathrm{n}^{\circ} 1\right) \rightarrow$. Mauléon, 59 x 44 puis 56 × $45 \mathrm{~cm}$ Titre basque à partir de janv. 1985 : Ziberoko bizia. - En français; quelques textes basques

* Morsure! Irrégulier. [nov./déc. 1979 - avr. 1981] (9 nº). - [Bayonne], 30 x $21 \mathrm{~cm}$ En français. - Rédigé par des élèves du Lycée René Cassin [Bayonne]

Mot (Le) de Jean Espilondo, votre conseiller général voir Lettre (La) de Jean Espilondo,

Mot (Le) du maire [Ordiarp] voir Ordiarp. Le mot du maire

* Mouguerre. Mugerreko berriak... Lettre d'informations municipales de la commune de Mouguerre. Irrégulier. oct. $2001\left(\mathrm{n}^{\circ} 1\right) \rightarrow$. Mouguerre, 30 x $21 \mathrm{~cm}$

En français. - Suite de: Bulletin d'information municipal de Mouguerre

Mouguerre. Bulletin municipal voir Egun eta bihar

Mouguerre, les trois clochers. Mouguerre-bourg, Mouguerre-port, MouguerreEliçaberry. Trimestriel. [...]. - Mouguerre, 21 x $14 \mathrm{~cm}$

Bulletin paroissial. - Paraissait en 1966 et 1967. - En français. - Texte en partie identique à celui de nombreux autres bulletins paroissiaux, impr. à Belley 
Moulin (Le) à paroles. Magazine publié par l'Ecole publique de Villefranque. $2 n^{\circ}$ par an puis irrégulier. mars 1998 - juin 2000 (n॰[1]-6). avril $2001 \rightarrow$. [Villefranque], 20 x $21 \mathrm{~cm}$ En français

* Mouton (Le) fonceur. [1986] - [...]. - Anglet, 30 x $21 \mathrm{~cm}$

En français. - Rédigé par des élèves du Collège Endarra [Anglet]

Mouvement pour la France... voir M. P. F.

Mugertarra. Bulletin bilingue de l'association culturelle et sportive "Mugertarrak». Trimestriel. automne 1978 - automne $1979\left(\mathrm{n}^{\circ} 1-4\right)$. - [Mouguerre], $30 \times 21 \mathrm{~cm}$ En basque et français

Mundial-infos. La lettre quotidienne du championnat du monde professionnel de cesta punta. 12 - 23 sept. 2001 (n`1-11). - Biarritz, $30 \times 21 \mathrm{~cm}$

Avec un $n^{\circ} 0$ du 25 août 2001. - En français; quelques textes basques. - A paru durant le 11e championnat du monde; publ. par le comité d'organisation

Muni-biblio-Z.U.P. Bulletin mensuel d'informations [puis d'informations et d'animation] de la Bibliothèque municipale, Annexe des Hauts de Sainte-Croix. Irrégulier. $1^{\text {er }}$ oct. 1976 15 févr. 1977 (n¹-6). juin 1977 - mai 1978 (4 n) [?]. - Bayonne, $21 \times 14 \mathrm{~cm}$

Recueil de textes et de dessins d'enfants, impr. par l'Imprimerie spéciale de La Ronde; jusqu'en févr. 1977 suppl. de: Ronde (La); de juin 1977 à mai 1978 en constitue les n²5 à 28. - A partir de juin 1977 porte en tête: Ville de Bayonne. - En français

63 Muscade. \{Le bulletin de l'amitié en poésie\}. Trimestriel ou irrégulier. [mai 1977 - 1988] ([1 re $]-11^{\mathrm{e}}$ année, $\left.\mathrm{n}^{\circ}[1]-30\right)$. - Anglet puis Bayonne, $30 \times 21$ puis $21 \times 15 \mathrm{~cm}$

En français. - Dir. Jean-Pierre Rasse. - Fondé par un groupe de collaborateurs de: Vents et marées, après leur départ de cette revue. - Le n¹5 (hiver 1981) a donné lieu à 2 éd. différentes

4 Musée Bonnat, Ville de Bayonne [puis Musée (Le) Bonnat]. \{Informations\}. Semestriel puis annuel. [1983] - oct. 1989/ juin 1990 (n¹-10). juin 1992 - 1993/ 94 (3 nº). - Bayonne, 44 $\times 29 \mathrm{~cm}$

En français

Musée historique de Biarritz. Etudes et documents. Publication de l'Association des amis du Vieux Biarritz. déc. 1994 - déc. 1995 (n¹-2). déc. 2001 (n³). - Biarritz, $30 \times 21 \mathrm{~cm}$ En français

\section{INDEX}

Index chronologique : 20e siècle

Index géographique : Pays basque (France)

Thèmes : bibliographie

Mots-clés : publication périodique 